\title{
LAS VOCES DE LOS DÁLIT EN RADIO COMUNITARIA: CONTRADICCIÓN ENTRE EL PRINCIPIO Y LA PRÁCTICA EN EL SUR DE ASIA
}

\section{VOICES OF 'DALIT' IN COMMUNITY RADIO:} CONTRADICTION IN PRINCIPLE AND PRACTICE IN SOUTH ASIA

J. B. Biswokarma Investigador y periodista independiente jb.sangidagmail.com radicado en Nepal. Autor del libro Los
dálit en los medios nepaleses: participació dálit en los medios nepaleses: participació
y contenidos, el único que trata el tema en Nepal. Ha escrito y publicado diversos artículos sobre los dálit, su trasformación sociopolítica, reestructuración de Esta inclusion y reforma agraria, entre otros. Traducción de Alba Trouchon (mayolta@gmail.com).

\section{Resumen Abstract}

No hay una sola definición de radio There is no single definition of Community comunitaria. Esta ha sido definida como un Radio. Community radio has been defined medio que pertenece a las personas, pues as a radio for people, own, operated and es operada y controlada por ellas y para controlled by people. UNESCO has defined ellas. UNESCO la define como "un medio community radio as "a medium that gives que provee de voz a quienes no la tienen, que sirve de portavoz a los marginados y se encuentra en el núcleo de los procesos comunicativos y democráticos de las sociedades".

Palabras clave Radio comunitaria, dálit, sur de Asia. voice to the voiceless that serves as a

mouthpiece of the marginalised and is a

the heart of communication and democratic process within societies”.

Keywords

Community Radio, Dalit, South Asia. 


\section{Contexto}

No hay una sola definición de radio comunitaria. Esta ha sido definida como un medio que pertenece a las personas, pues es operada y controlada por ellas y para ellas. UNESCO la define como "un medio que provee de voz a defie como un moz quienes nola tienen, que sirve de portavoz a los marginados y se encuentra en el núcleo de los procesos comunicativos y democráticos de las sociedades" (UNESCO, 2001). Tal como lo define la Asociación Mundial de Radios Comunitarias (AMARC):

La filosofía histórica de la radio comunitaria consiste en emplear este medio como la voz de los sin voz como portavoz de los pueblos oprimidos (sea por motivos relacionados con la radio misma, con géneroocon clase) yengeneral ma, con género o con clase) y en genera como un mecanismo de desarrollo. La radio comunitaria reúne tres aspectos principales: una finalidad no lucrativa, propiedad y control por parte de la comunidad y participación comunitaria (A resource guide, AMARC, 1998).

También se le conoce como radio local, radio del pueblo, radio democrática entre otros, y se define como entidad autónoma cuya supervivencia, libre de cualquier objetivo o meta vivencia, chre de culquier objetivo o meta comercial, depende de la comunidad. Las de finiciones de radio comunitaria resaltan su responsabilidad frente a los sectores marginados en la sociedad. De hecho, los dálit son marginados, en gran medida, en los países del sur de Asia, por tanto, deben ser prioritarios en la agenda de las radios comunales.

Aproximadamente doscientos sesenta millones de personas se conocen como dálit, en un mundo que enfrenta discriminación por razones de casta. Los dálit representan cerca de un 16 por ciento de la población en India, un 13 por ciento en Nepal, así como un número significativo en otros países del sur de Asia. La sociales discriminator tanto en las esfera pública como en la privada, ha orignado viopubica como en la privada, ha originado vioaciones flagrantes y negación sistémica de los derechos humanos de aquellas personas nacidas como "intocables", "sin casta", en las "castas inferiores”. La comunidad dálit es un grupo como parte de la vida diaria, manifestada en prácticas inhumanas como la intocabilidad. El sistema de castas se basa en un orden jerárquico cuyos grupos sociales se distinguen de otros de acuerdo con su grado de pureza y posición social dentro del sistema feudal de castas hindúes (Biswokarma, 2013). Debido a las normas discriminatorias, sistema de valores creado por los líderes hindúes, descle hace miles de años, los dálit enfrentan exclusión política privación económica y discriminación sociocultural, en particular, dentro de la sociedad hindú. Los entes estatales de toma de decisiones del Estado cuentan entre sus miembros con una escasa representación de los dálit, al igual que los partidos políticos. La política del sur de Asia se rige por las decisiones de las denominadas castas superiores hindúes, o grupos de castas dirigentes, lo que ha dejado a los dálit sin voz y sin poder. En la toma de decisiones estatales, la representación nominal ha originado vacíos en la formulación de políticas y diseño de programas para la emancipación de la comunidad dálit.

A pesar de ser una comunidad laboriosa, los dálit son excluidos de los medios, incluidos la tierra y otros recursos naturales. Su pobreza imposibilita la consecución de buenas oportunidades en educación, lo que al originar desempleo, perpetúa su situación de escasez. El sistema de trabajo basado en la explotación aún existe en India, Nepal y otros países afec- tribuido a la pobreza. Incluso los dálit han tratado de probar que son seres humanos. La intocabilidad aún persiste en la sociedad, también en lugares públicos. La constitución y las disposiciones legales en Nepal e India rechazan la discriminación con base en la casta, mientras que esta se lleva a la práctica en la vida social (Aahuti, 2004). Las denominadas castas superiores controlan las instituciones administrativas y judiciales. En su mayoría, estas tienen una mentalidad discriminatoria y prejuiciada en contra de los dálit. La policía no registra con facilidad los casos de intocabilidad y presiona por una solución negociada con el responsable. La estructura administrativa y judicial tampoco es incluyente y muchas de las denominadas autoridades tienen prejuicios contra los dálit que suelen verse reflejados en los procesos judiciales. Dicha comunidad no está recibiendo justicia ni por vías sociales, administrativas 0 judiciales. Los dalit no cuentan con una voz dentro de los difejudiciales. Los dát no cuentan con una voz dentro de liferentes sectores del estado como institución social, lo que se ha visto reflejado en los medios de difusión de los países del sur de Asia.

La comunidad dálit ha luchado por justicia desde hace ya bastante tiempo. El Dr. Ambedker ha jugado un papel crucial en el manejo de la discriminación con base en la casta en India. En Nepal, los dálit también han mantenido su búsqueda de justicia por setenta años. Como resultado, el movimiento de los dálit (conocidos como "Castas Reconocidas" en India) ha alcanzado algunos logros significativos. Para el caso de Nepal e India, el Estado se ha manifestado a través de organizaciones gubernamentales, al crear mecanismos estatales para tratar con la situación, tales como la Comisión Nacional Dálit (NDC). Si bien su papel no es lo suficientemente efectivo, la situación de los dálit se ha convertido en un asunto importante dentro de la agenda política, en particular en Nepal e India. La constitución de Nepal e India rechaza la intocabilidad, mientras que el gran vacío en su implementación de las disposiciones legales se ve reflejado en la exclusión de la comunidad dálit por parte de los entes de toma de decisiones y los organismos judiciales. En la actualidad, el movimiento dálit es internacional y su agenda se ha ampliado en el foro de las Naciones Unidas, así como en el Parlamento Europeo. En Nepal, la noción de inclusión se ha tornado prominente, a la vez que ha sido incluida en contextos constitucionales y políticos. No hay un partido político, Estado o sociedad civil en Nepal que simplemente ignore este punto en su agenda. 


\section{La radio comunitaria en la promoción de los derechos de los dálit}

$\mathrm{Al}$ actuar como importantes catalizadores de cambio político, los medios han desempeñado un papel significativo dentro del movimiento democrático en Nepal. Una cantidad aproximada de 250 radios comunales opera en Nepal, las cuales han ejercido una función muy importante en la entrega de información y poder a los ciudadanos en niveles populares. Sobre todo, las radios comunitarias han adoptado el principio de participación de la comunidad dálit en sus juntas directivas, noticias y programas, que aún no se implementa de manera global.

munidad, debe convocar al Estado para poner fin a sus problemas y facilitar a los dálit el disfrute de sus derechos democráticos. También debe sensibilizar a aquellos fuera de la comunidad dálit y llamarlos a erradicar la discrimnación con base en la casta y la intocabilidad. La radio comunitaria provee espacios de discusión de diferentes aspectos sociales con el objetivo de mejorar la vida de las personas.

También se reconoce que el primer objetivo de la radio comunitaria es la promoción de la democracia, la creación de un discurso entre comunidades y la provisión de oportunidades $\begin{array}{ll}\text { Los medios nepaleses han ejercido un rol im- } & \text { de encuentro entre diferentes puntos de vista. } \\ \text { portante en la denuncia de los casos de viola- } & \text { En apariencia, las voces de los grupos margi- }\end{array}$ ción de derechos humanos y en el cubrimiento nados, en particular las voces de los dálit, no se de los casos de discriminación. Sobre la base escuchan en los países del sur de Asia, a través de tales noticias, los activistas y las partes inte- de la radio comunitaria. La radio comunitaria resadas siguen luchando por justicia. Sin em- no ha dado prioridad a los asuntos relacionabargo, la proporción de tales noticias es peque- dos con los dálit en los programas y en las notiña en comparación con la de sus problemas. cias. La contradicción entre principio y práctiLos medios nepaleses, incluyendo a las radios ca en las radios comunitarias es evidente.

comunitarias, se han dedicado a cubrir los

eventos y los casos de discriminación y viola- Si creemos en la noción comunicativa según ción de derechos humanos, antes que a inda- la cual solo las personas informadas pueden gar las raices y causas genuinas de exclusión tomar decisiones correctas, en primer lugar, contra la comunidad dálit. Las investigaciones la radio comunitaria debe contribuir a que las han demostrado que las aspiraciones reales de personas se apropien de sus derechos, y en selos dálit no son una prioridad para las radios gundo lugar, debe empoderar a la población, comunitarias en Nepal, al igual que en India a través de entrega de información adecuada y en otros países de Asia meridional. Por prin- que les permita disfrutar de sus derechos. La cipio, las radios comunitarias se rigen por su radio comunitaria puede y debe desempeñar compromiso con las poblaciones marginadas, un papel crucial en la denuncia de cuestiones ciones comerciales. Las radios comunitarias públicam y se encuentren maneras de deben ser responsables en la denuncia de si- traspasar las barreras sociales. Fundamentuaciones propias de poblaciones marginadas, talmente, las radios comunitarias deben amtales como los dálit. El discurso debe expresar pliar el acceso a la información y educar a las las dificultades por las que atraviesa dicha co- personas a través de la facilitación y entrega de información que les permita cam prejuicia valores discriminatorios de sistema de castas. Tal como sostienen los principios de comunicación para el cambio de comportamiento, las radios comunitarias pueden ejercer una función fundamental en la reducción de práctic en la reducción de prácticas inhumanas en contra de la comunidad dálit, tales com la intocabilidad, exclusión, explotación laboral y dominación. Por tanto, el rol de la radio comunitaria en la erradicación de la discriminación con base en la casta esc

Además, la diversidad social en términos de casta, etnicidad, idioma y género, entre otros, deben verse reflejado tanto en los contenidos como en la estructura de la radio comunitaria.
(Pringle y Subba 2007) sostiene que la élite ha capturado la radio comunitaria, de manera que, en particular, hay poca representación de los grupos comunales en cuanto se refiere a género, casta y grupos étnicos, a nivel de toma de decisiones y diseño de políticas. En especial, minorías tales como los dálit no cuentan con un fácil acceso a la información y a la participación dentro de las instituciones mediáticas. Para el caso de Nepal, los dálit constituyen apenas 1.68 por ciento del número total de los periodistas nepaleses. Los lineamientos de las radios comunitarias de una estructura y la entrega de una estructura y la entrega de información integradora. Esto significa que la participación de las personas marginadas en la programación debe ser prioritc la práctica. Otro estudio ha demostrado que solo el 7 por ciento de las noticias y de la programación y el 1.84 por ciento de las operaciones de los subcomités poseen representación de miembros de la comunidad dálit (Biswokarma, 2007). En las radios come, 2007). En las radios corunitarias de India y

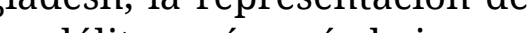
los dálit es aún más baja que en Nepal. Sin embargo, a través del discurso propio de las radios comunitarias, se han logrado expresar políticas, conich nivel de política hasta uno de mplementación, lo cual representa una iniciativa para la inclusión de los dálit en la radio comunitaria.

Tabla 1: Participación de los dálit en la radio comunitaria en Nepal.

\begin{tabular}{|c|c|c|c|c|}
\hline S.N. & Descripción & $\begin{array}{c}\text { Total de } \\
\text { estaciones } \\
\text { comunitarias }\end{array}$ & Dálit & \\
\hline 1. & Comité ejecutivo & 238 & 17 & $7 \%$ \\
\hline 2. & $\begin{array}{c}\text { Liderazgo en salas de redacción } \\
\text { y programación }\end{array}$ & 204 & 13 & $6 \%$ \\
\hline 3. & Sub-comité de operaciones & 163 & 3 & $1.84 \%$ \\
\hline
\end{tabular}

Fuente: J.B. Biswokarma, 2007. 
Existe el riesgo de que la orientación comunitaria y el énfasis en la programación de interés público se debiliten. Otro estudio (Biswokarma, 2007) sobre la radio comunitaria en Nepal ha demostrado que en promedio solo el 0.06 por ciento del espacio radial es adjudicado a estos temas en una estación de radio que transmita durante dieciocho horas por día. Las radios comunitarias tratan muy pocos asuntos relacionados con los dálit en las noticias y programas por igual, y el espacio asignado no es justificación para la responsabilidad de la radio comunitaria con la población dálit.

Otro rasgo importante de la radio comunitaria es la creación de diversidad en su programación, que permita que la voz de los excluidos y de los marginados sea escuchada, que promueva el diálogo abierto, el proceso democrático, el desarrollo y la transformación social, la buena gobernanza y sirva de voz de quienes no la tienen. Pringle y Subba (2007) también recomiendan a las radios comunitarias dar prioridad a los contenidos de grupos excluidos.

Las radios comunitarias son considerados un agente de transformación social, porque pueden llegar a desempeñar un papel significativo en la trasformación de la actitud y comportamiento de las personas desde la rigidez hasta la modernidad y humanidad. La personas desde la rigidez hasta la modernidad y humanidad. La
radio comunitaria logra crear valores alternativos con base en la radio comunitaria logra crear valores alternativos con base en la
humanidad y en la dignidad, en lugar de los valores tradicionales discriminatorios, a la vez que provee información adecuada al público. También crea discurso comunitario al entregar información de forma creativa en la búsqueda de la constitución de una sociedad humanitaria, donde la discriminación en relación con la casta no exista.

La Asociación de Radios Comunitarias en el Sur de Asia, así como las estaciones individuales de radio, se encuentran fundamentalmente de acuerdo con dar prioridad al contenido de los grupos marginados de la sociedad, incluyendo a la comunidad dálit, por medio de la elaboración de políticas inclusivas para sus estaciones de radio; esto no se refleja en la práctica aún. Hay una inmensa brecha y contradicción entre principio y prácticas en las radios comunitarias en Asia meridional.

¿Es tarde entonces para monitorear las políticas, participación y contenidos de las estaciones de radio comunales desde la perspectiva dálit, con el fin de descubrir si están siguiendo en realidad los principios fundamentales de la radio comunitaria?

\section{Intentos de las radios comunitarias por hacer escuchar las voces de los dálit}

En relación con la situación dálit, el discurso que se ha iniciado a nivel surasiático en los medios comunitarios es un buen comienzo. Las organizaciones centrales de radiodifusoras comunitarias tales como la Asociacion de difusoras Comunitarias (ACORAB) en Nepal, la Asociación de Radio Comunitaria en India, la Red de ONG para Radio y Comunicación de Bangladesh (BNNRC) y el Foro de Radios Comunitarias en India, entre otros, han iniciado una discusión sobre la participación de los dálit en la radio comunal y la producción de contenidos sobre su situación.

En algunos encuentros sobre la radio comunitaria, se han realizado declaraciones para asegurar la representación y otorgar prioridad a los contenidos de los dálit. En la declaración de Bangalore de 2010, claramente se sostiene: "crear espacios en las ondas sostiene: ccrear espacios en las ondas radiales para voces diversas y marginadas, sin distinción por razón su casta, credo raza, color, género, sexualidad, fe, discapacidades u otras diferencias". Otro encuentro se celebró entre las partes interesadas y los representantes de ministerios involucrados en 2013, en KatKatmandú de 2013, también se sostiene que

[...]se protege y promueve la libertad de expresión, especialentre grupos marginados que incluyan mujeres, pobres en zonas urbanas y rurales, minorías sexuales, discapacitados, dá- lit, las comunidades indígenas e inmigrantes, y se hace un llamado a la radio comunitaria en el sur de Asia a tornarse más integradora en términos de participación, manejo y propiedad, en especial en lo que se refiere a las mujeres, las minorias sexuates, los dálity las piedacit y las poblaciones indigenas y a hacerse cargo de una campaña concertada a través de radio comunitarias en contra de la intocabilidad la discriminación.

En materia de participación y contenidos, la Declaración de Corea de 2013 también ha conservado el espíritu de una radio comunitaria incluyente. En las declaraciones, se cita:

[...] consideramos que entre algunos de los países miembros, existen comunidades indígenas que son marginadas y a quienes se les niega el acceso a los derechos humanos básicos, y que entre algunos de los países miembros, a las mujeres, a las personas de diversa orientación sexual e identidad de género, a los discapacitados, a las comunilas religiosas y etnicas se les margina y se les niega derechos humanos básicos. Las radios comunitarias reconocen ser una herramienta importante de destrucción de barreras sociales y estereotipos que marginen o excluyan a las personas en materia de género, raza, etnicidad, orientación sexual dentidad de género, clase, casta creencias religiosas o discapacidad. Además, deciden crear espacios en las ondas radiales para las voces diversas y marginadas, sin hacer caso de su de género, raza, etnicidad, orientación sexual e identidad de género, clase, casta, creencias religiosas o discapacidad. 
Algunas reflexiones sobre la declaración pueden verse en el contexto nepalés. La organización central de radio comunitaria ACORAB ha empezado a hacer su estructura más inclusiva. Un vicepresidente y una radio comunitaria central se encuentran representados en el comité central de ACORAB. De manera similar, el plan estratégico a cinco años de ACORAB está orientado a inide ACORAB. De manera similar, el plan estratégico a cinco años de ACORAB está orientado a ini-
ciar una campaña en contra de la discriminación con base en la casta. De hecho, una iniciativa conjunta de Jagaran Media Center, ACORAB y AMARC es denominada las "Radios Comunitarias en contra de la discriminación con base en la casta en Nepal”. Es una buena iniciativa, que esperamos sirva de apoyo a las voces de dálit en la esfera pública, y que debe intentarse en otros países del sur de Asia.

\section{Logros hasta} el momento

Hasta el momento, en materia de radio comunitaria, pueden concluirse cinco clases de logros para la comunidad dálit. En primer lugar y principalmente, las radios comunitarias aceptan la agenda dálit como una cuestión de importancia que merece atención y trataquento La mayoŕa de tas ramiento. La mayoría de las radios comunitarias han contrbuido a un aumento de la con-
ciencia y sensibilización sobre la importancia de participación y contenidos significativos para los dálit en las radios comun tarias. En segundo lugar, las participaciones de los dálit en las radios comunitarias han incrementado. En comparación con otras instituciones mediáticas, la representación en radios comunitarias es notable si bien no lo suficientemente influyente, con una representación en juntas directivas, tación en juntas directivas, noticias y programas, lo que Para el caso de Nepal, diez de doscientos cincuenta y tres reclamo de voces incluyentes directores radiales provienen en los países del sur de Asia y de la comunidad dálit. Ocho la sensibilización de las partes de diez directores radiales dá- interesadas. Tal fomento se lit aún conservan su posición. refleja en una presión moral La misma comunidad dálit ha por el diseño de lineamientos empezado a operar la radio específicos que den prioridad comunitaria; dos de tales esta- a la situación de los dálit denciones operan en Nepal. tro de los contenidos radiales. En cuarto lugar, las radios comunitarias han facilitado el acceso de las comunidades dálit a información relacionada con varios aspectos que son relevantes en su vida diaria. Tal acceso a la información, les ha facilitado el disfrutar de sus derechos democráticos. cos. Desde la perspectiva del movimiento dálit, las radios comunitarias han apoyado su movimiento en el sur de Asia al denunciar las preocupaciones de la comunidad dálit y al sensibilizar a las personas fuera de dicho grupo. Por último, el dichovimiento de las ratimo, el movimiento de las radios comunitarias en el sur de Asia ha otorgado importancia a la situación de los dálit y ha dado prioridad en su agenda a una campaña colectiva en ascenso en contra de la discriminación con base en la casta y a la intocabilidad. Esto puede conducir a las radios comunitarias en el sur de Asia y a sus contenidos a ser más incluyentes.

\section{Retos existentes}

Desde la perspectiva de los dálit en los países del sur de Asia, aún existen contradicciones entre principio y práctica en las radios comunitarias. En relación con la radio comunitaria, los lineamientos y casi todos los documentos han aceptado en sus principios la noción de inclusión en estructura y contenidos sin que se vean reflejados en la práctica. Hasta cierto punto, la radio comunitaria ha tratado de situar a los dálit en posiciones de liderazgo que in de liderazgo que incluyen los comités de dirección, pero muchas de ellas parecen más muestras de aprecio o exhibiciones, ya que en muchos no se ejerce un papel decisorio en la elaboración de políticas y programas en las estaciones. La transformación de la tradicional mentalidad discriminatoria hacia los dálit, así como otros aspectos, son aún retos que los países en el sur de Asia deben enfrentar. Las políticas en inclusión de los dálit que se buscan implementar aún representan un reto, ya que muchas de las denominadas castas superiores no confían en ellos, a pesar de su alta competencia. En definitiva, la radio comunitaria debe tratar la situación dálit como prioritaria y principal motivo de preocupación, con el fin de facilitar el tratamiento de sus problemas, y de hacer de la proactividad una característica necesaria de la radio comunitaria.

Un rasgo importante de la radio comunitaria es su función de ser la voz de quienes no la tienen dentro de la sociedad. Sin embargo, las radios comunitarias en los países del sur de Asia no bargo, las radios comunitarias en los paises del sur de Asia no han llevado a cabo ninguna estrategia específica para erradicar la discriminación con base en la casta como misión fundamen-
tal. Se ha dado incluso lugar a criticismos que sostienen que las radios comunitarias tienden a la discriminación de espacios dedicados a la agenda dálit y de las personas marginadas en la sociedad. Para enfrentar este reto, la radio comunitaria debe 作 ras a representar las voces y aspiraciones de tales comunidades.

No existe un mecanismo independiente para supervisar los contenidos de la radio comunitaria en Nepal, así como en otros países del sur de Asia. Es importante observar la compatibilidad de los contenidos en relación con sus agendas prioritarias y asignar espacios a las minorías locales, incluyendo a los dálit.

\section{Conclusión}

Hay interrogantes fundamentales que las radios comunitarias deben responder. Debe realizarse realizare una revison sobre la base de los rasgos principaes de las radios comunitarias. $¿$ ¿Han conservado las radios comunitarias los estándares mínimos en materia de participación y contenidos? ¿Sienten los oyentes la radio comunitaria como propla? ¿Qué ique factores han influido en la falta de inclusión de nuestras estaciones radiales desde la perspectiva dálit y los grupos excluidos? ¿Qué estrategias deben llevarse a cabo para combatir dicho problema? También, planes y estrategias periódicas deben diseñarse con el propósito de erradicar prácticas inhumanas tales como la intocabilidad en las sociedades del sur de Asia.

Además, el fomento continuo de la formulación de políticas incluyentes en los medios comunitarios es esencial para el levantamiento de las voces reales de las comunidades marginadas y el fortalecimiento de la democracia. Para promover humanidad y crear armonía social, las radios comunitarias deben seguir una hoja de ruta específica, así 
como políticas y lineamientos definidos que deben implementarse, con el fin de erradicar la intocabilidad y la discriminación con base en la casta. En consideración del hecho que la sociedad solo puede ser democrática cuando la población disfruta de sus derechos con libertad, la radio comunitaria debe contribuir a tal democracia.

\section{Referencias}

Aahuti. (2004). Hindu Samajma Dalit Jatiya Mukriko Prashna (A question regarding Dalit in Hindu Society). Nepalko Sandarbhama Samajshastriya Chintan. Mery Des Chene Eds, pp. 475-521. Lalitpur: Social Science Baha.

Biswokarma. (2007). Dalit in Community Radio: Policy, Leadership and Programmes. An unpublished research report.

Biswokarma. (2013). Nepali Mediama Dalit: Sahabhagita ra Bisayabastu (Dalit in Nepali Media: Participation and Contents). Devraj Humagain and Bimal Acharya eds. Kathmandu: Martin Chautari.

Caste Based Discrimination in South Asia. (2009). Situation, Overview, Responses and Ways forward. Denmark: International Dalit Solidarity Network (IDSN).

Pringle, Ian y Bikram Subba. (2007). Ten Years on: The State of Community Radio in Nepal. Unpublished Report. Recuperado de: http://portal.unesco.org/geography/ en/files/7991/11966615215State of Community Radio in Nepal_abridged.pdf/ State\%2Bof\%2BCommunity\%2BRadio\%2Bin\%2BNepal_abridged.pdf

AMARC. (1998). What is Community Radio: A Resource Guide. AMARC Africa and Panos Southern Africain collaboration with IBIS/Interfund and WACC. Recuperado: http://www.amarc.org/ documents/manuals/What_is_CR_english.pdf

UNESCO. (2001). Community Radio Handbook. http://www.unesco.org/webworld/publications/ community_radio_handbook.pdf

1

1

\section{1}

\section{1}

\section{1}

1

\section{1}

